\title{
BMJ
}

\section{Effect of real-time feedback during cardiopulmonary resuscitation outside hospital: prospective, cluster- randomised trial}

\begin{abstract}
David Hostler, research associate professor of emergency medicine, ${ }^{1}$ Siobhan Everson-Stewart, research scientist, ${ }^{2}$ Thomas D Rea, associate professor of medicine, ${ }^{3}$ lan G Stiell, professor and chair of emergency medicine, ${ }^{4}$ Clifton W Callaway, associate professor and vice-chair of emergency medicine , ${ }^{1}$ Peter J Kudenchuk, professor of medicine, ${ }^{3}$ Gena K Sears, resuscitation outcomes consortium programme manager , ${ }^{2}$ Scott S Emerson, professor of biostatistics, ${ }^{2}$ Graham Nichol, professor of medicine ${ }^{2,3,5}$ and the Resuscitation Outcomes Consortium Investigators
\end{abstract}

\section{University of Pittsburgh, Department of Emergency Medicine, 3600 Forbes Ave, Suite 400A, Pittsburgh PA 15261, USA \\ ${ }^{2}$ University of Washington Clinical Trial Center, Department of Biostatistics, Seattle WA, USA \\ ${ }^{3}$ University of Washington, Department of Medicine, Seattle WA \\ ${ }^{4}$ University of Ottawa, Department of Emergency Medicine, Ottawa ON, Canada \\ ${ }^{5}$ University of Washington- Harborview Center for Prehospital Emergency Care, Seattle WA Correspondence to: $\mathrm{D}$ Hostler hostlerdp@upmc.edu}

Cite this as: $B M J$ 2011;342:d512 doi:10.1136/bmj.d512

\section{ABSTRACT}

Objective To investigate whether real-time audio and visual feedback during cardiopulmonary resuscitation outside hospital increases the proportion of subjects who achieved prehospital return of spontaneous circulation. Design A cluster-randomised trial.

Subjects 1586 people having cardiac arrest outside hospital in whom resuscitation was attempted by emergency medical services (771 procedures without feedback, 815 with feedback).

Setting Emergency medical services from three sites within the Resuscitation Outcomes Consortium in the United States and Canada.

Intervention Real-time audio and visual feedback on cardiopulmonary resuscitation (CPR) provided by the monitor-defibrillator.

Main outcome measure Prehospital return of spontaneous circulation after CPR.

Results Baseline patient and emergency medical service characteristics did not differ between groups. Emergency medical services muted the audible feedback in $14 \%$ of cases during the period with feedback. Compared with CPR clusters lacking feedback, clusters assigned to feedback were associated with increased proportion of time in which chest compressions were provided (64\% $\mathrm{V}$ $66 \%$, cluster-adjusted difference $1.9(95 \% \mathrm{Cl} 0.4$ to 3.4$)$ ), increased compression depth (38 v $40 \mathrm{~mm}$, adjusted difference 1.6 ( 0.5 to 2.7$)$ ), and decreased proportion of compressions with incomplete release $(15 \%$ v $10 \%$, adjusted difference $-3.4(-5.2$ to -1.5$))$. However, frequency of prehospital return of spontaneous circulation did not differ according to feedback status ( $45 \%$ v $44 \%$, adjusted difference $0.1 \%$ ( $-4.4 \%$ to $4.6 \%)$ ), nor did the presence of a pulse at hospital arrival $(32 \% \mathrm{~V}$ $32 \%$, adjusted difference -0.8 ( -4.9 to 3.4$)$ ), survival to discharge $(12 \% \vee 11 \%$, adjusted difference -1.5 ( -3.9 to $0.9))$, or awake at hospital discharge $(10 \% \vee 10 \%$, adjusted difference -0.2 (-2.5 to 2.1$)$ ).
Conclusions Real-time visual and audible feedback during CPR altered performance to more closely conform with guidelines. However, these changes in CPR performance were not associated with improvements in return of spontaneous circulation or other clinical outcomes.

Trial Registration Clinical Trials NCT00539539

\section{INTRODUCTION}

Cardiopulmonary resuscitation (CPR) is an essential link in the chain of survival for treating cardiac arrest. However, performance of cardiopulmonary resuscitation is highly variable both outside hospital and in hospital. ${ }^{12}$ Interruptions in chest compression, inadequate depth of chest compression, and high rates of ventilation adversely affect blood flow during chest compressions and can hinder resuscitation. ${ }^{34}$ Suboptimal CPR, particularly time spent without chest compressions (low chest compression fraction), can reduce survival of cardiac arrest patients. ${ }^{5}$

Current technology incorporated into a monitordefibrillator can assess core components of CPR (rate and depth of compressions, extent of decompression, and timing of ventilations) through the use of an accelerometer and impedance changes across the defibrillation electrodes. This technology can also provide realtime audiovisual feedback so that the rescuer is prompted to perform according to guideline specifications. Use of such feedback increases the likelihood of performing CPR in accordance with guidelines during training and simulation. ${ }^{6}$ Providing real-time feedback enables more consistent CPR performance both in and outside hospital. ${ }^{78}$ Although real-time CPR feedback is a promising strategy to improve resuscitation care, little is known about the clinical effects of feedback during resuscitation. Prior studies have not been designed to rigorously assess the clinical outcome effects of realtime CPR feedback. 
Three sites within the Resuscitation Outcomes Consortium conducted a prospective cluster-randomised trial to assess the clinical effects of real-time CPR feedback during emergency medical services' attempted resuscitation of prehospital cardiac arrest. We hypothesised that the feedback would increase the proportion of patients with return of spontaneous circulation compared with resuscitation without such feedback.

\section{METHODS}

Design and setting

The Resuscitation Outcomes Consortium was formed from multiple sites in the United States and Canada to conduct controlled trials of prehospital treatment of cardiac arrest and life threatening trauma. This cluster-randomised controlled trial compared standard CPR with CPR with real-time feedback in selected emergency medical services participating in the Resuscitation Outcomes Consortium. Data collection was accomplished in two US sites (King County Washington, Pittsburgh, and Westmoreland County, Pennsylvania) and one Canadian site (Thunder Bay, Ontario) through the existing infrastructure of Epistry-Cardiac Arrest. The Epistry-Cardiac Arrest is a prospective multicentre observational registry of cardiac arrests outside hospital that are attended by emergency medical services and receiving institutions that participate in the Resuscitation Outcomes Consortium clinical research network. ${ }^{910}$ About half of the subjects in this trial were concomitantly enrolled in the Resuscitation Outcomes Consortium PRIMED randomised clinical trial. ${ }^{112}$ The study was approved by the local institutional review or research ethics boards for each participating agency, with waiver of informed consent under minimal risk criteria. The first subject was enrolled on 1 February 2007, and enrolment ended on 9 March 2009 after reaching full enrolment.

\section{Population}

The study subjects were adults aged $\geq 20$ years who had a cardiac arrest outside hospital and received rescue shocks or chest compressions by a participating emergency medical service with a monitor-defibrillator capable of real-time CPR feedback. Subjects were excluded if known to be prisoners or pregnant, if the cardiac arrest was due to traumatic causes, or if the subject had a "do not resuscitate" order.

\section{Intervention and randomisation}

CPR feedback was provided through proprietary QCPR software operating in the Philips MRx monitordefibrillator (Philips Medical Systems, Andover MA, USA). The manufacturer provided participating emergency medical services with commercially available devices equipped with visual and audible real-time feedback on quality of CPR. The defibrillator includes a "puck" with force transducer and accelerometer that is placed on the patient's sternum and connected to the monitor to measure the frequency, depth, and release of chest compressions. Ventilation is measured by means of changes in transthoracic impedance between the defibrillation electrodes or by capnography when this was used. Emergency medical service providers were trained in a manner consistent with local policy and standards, with the exception that the "puck" was to be used for data collection during resuscitation regardless of whether the CPR was randomised to provide feedback ("feedback-on") or not ("feedback-off").

The feedback feature of the defibrillator includes audible voice prompts and visual messages on the monitor screen that are triggered when measured chest compressions or ventilation deviate from guidelines or are interrupted. Providers can mute the voice prompts, but the visual prompts displayed on the monitor screen continue and an intermittent audible tone alerts the provider to the on-screen message. These feedback features can be activated or deactivated by an administrator password that was not available to the providers.

For this study, all the participating emergency medical agencies conducted a three month run-in period with the feedback feature turned off to establish familiarity with the use of the puck and with downloading data. After this period, a statistician in the coordinating centre randomised clusters ranging in size from individual emergency medical vehicles to groups of emergency medical agencies to feedback-on or feedbackoff. Each cluster remained in its assigned mode for two to seven months, after which it switched to the opposite treatment arm. At the end of those two treatment periods, each cluster was again randomly assigned to feedback-on or feedback-off. This cycle continued for the duration of the study. Each cluster switched treatment arms at least once, and up to four times, during the study.

\section{Data collection and definitions}

Data were abstracted from the emergency medical services' dispatch and patient care reports, hospital records, and the electronic defibrillator recordings. Standardised definitions and a uniform manual of operations were used to abstract patient characteristics (age, sex), circumstances of the cardiac arrest (witness status, arrest location, arrest before arrival of emergency medical service, presenting rhythm), prehospital care (bystander CPR, emergency medical service's response intervals), and outcome at hospital discharge. ${ }^{9}$ Initial presenting rhythm was classified as ventricular fibrillation or pulseless ventricular tachycardia, pulseless electrical activity, or asystole based on review of the emergency medical service's records and defibrillator electrocardiogram.

In some instances, the defibrillator was operated in automated external defibrillator mode, providing a shock or no shock, but the electrocardiogram was not available to classify the initial presenting rhythm. These cases, where a shock was delivered, were classified as ventricular fibrillation or pulseless ventricular tachycardia because of the high degree of specificity of the defibrillator for detecting shockable rhythms. ${ }^{13}$ 
Data about CPR performance were obtained from the electronic recordings downloaded from the defibrillators. This information was processed by the Philips Q-CPR software program, which displays a graphical and tabular summary of CPR processes to determine the minute-by-minute chest compression rate, chest compression fraction, ventilation rate, mean chest compression depth, and proportion of chest compressions with incomplete release. When CPR providers did not use the puck or if the accelerometer recording was unusable, we examined the transthoracic impedance channel recordings manually to calculate chest compression rate and fraction. Cases were checked by a study coordinator experienced in case review to assess if the automated measurements were accurate.

\section{Outcome measures and sample size estimates}

Restoration of spontaneous circulation before hospital admission was the primary study outcome and was defined as the presence of a palpable pulse in any vessel for any length of time during prehospital care. A pulse at hospital arrival was defined as the presence of a palpable pulse at the time of arrival to the emergency department.

Secondary outcomes included survival to hospital discharge, neurological status at discharge, and CPR variables. Survival to discharge was defined as discharge alive from hospital after the index arrest. Patients who were transferred to another acute care facility (for example, to undergo placement of an implantable cardioverter defibrillator) were considered to be still hospitalised. Patients transferred to a long term care facility or reclassified as a non-acute patient awaiting placement or chronic care were considered discharged. Neurological status at discharge was assessed by review of the medical records and was classified by degree of reported disability and discharge destination. A standardised instrument was used to categorise the neurological status of subjects at discharge according to disability.

This study of real-time feedback provided during prehospital CPR was designed with a power of $90 \%$ and a two sided alpha of 0.05 to detect a $10 \%$ absolute difference in return of spontaneous circulation between intervention and control arms, with an assumed 20\% incidence of return of spontaneous circulation at any time during resuscitation.

\section{Statistical analysis}

Baseline demographic information was summarised by treatment group using frequency (percentage) for categorical variables. For continuous variables, we report the median (interquartile range) and mean (standard deviation).

The primary analysis was a weighted, paired $t$ test of the rates of return of spontaneous circulation comparing periods of feedback-on versus feedback-off. Rates were paired by cluster; the cluster weights were proportional to the harmonic mean of the number of subjects treated within a cluster during the periods of feedback-on and feedback-off. ${ }^{14}$ Tables present the average within-cluster change, 95\% confidence interval for the change, and the respective $\mathrm{P}$ value. This analysis was repeated on the secondary outcomes of return of spontaneous circulation at time of arrival at

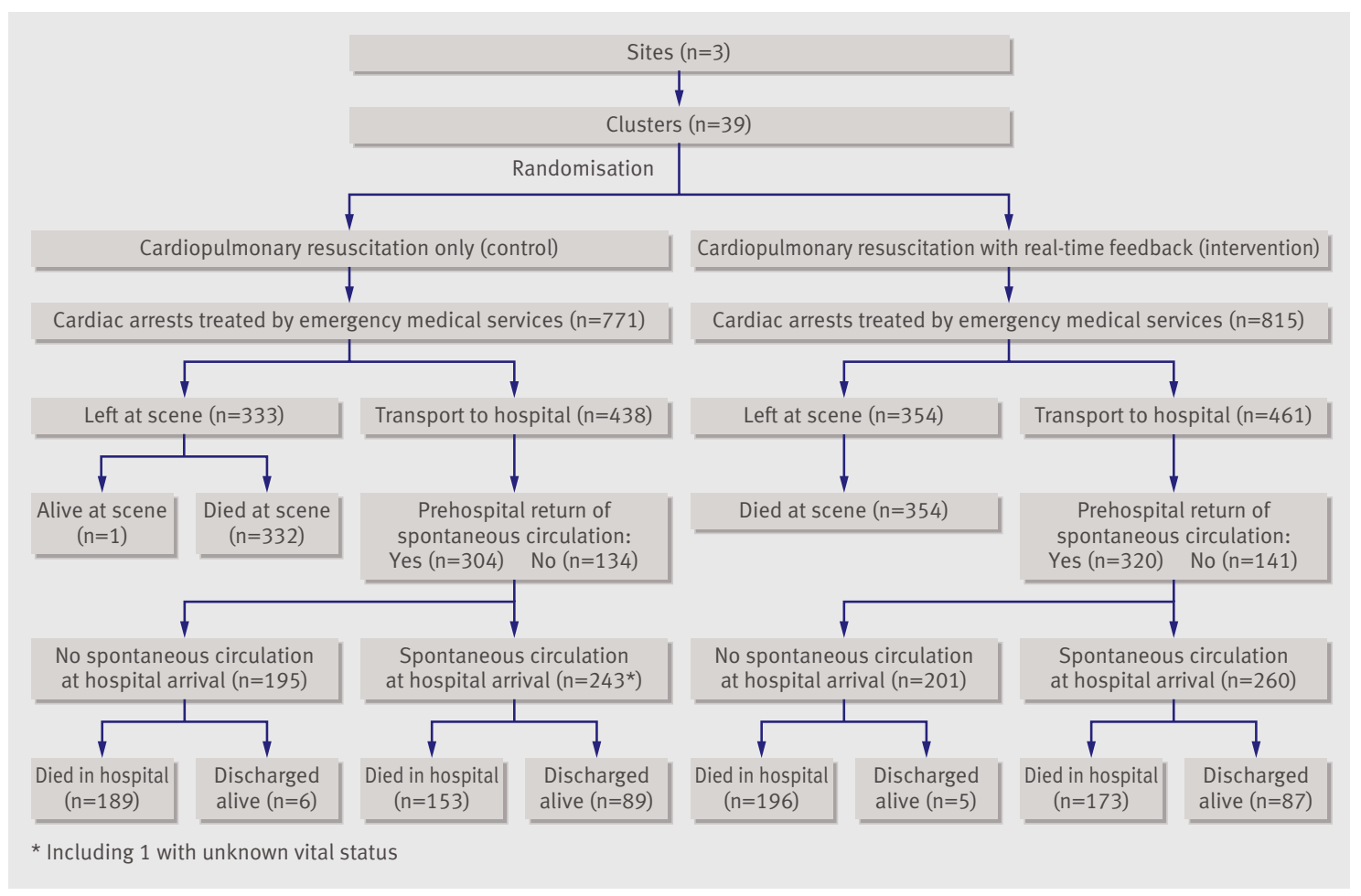


Table 1|Demographic and clinical characteristics of, and prehospital treatment received by, subjects with a cardiac arrest who received cardiopulmonary resuscitation (CPR) outside hospital: 233 patients during run-in phase of trial* and 1586 during evaluable period. Values are numbers (percentages) of patients unless stated otherwise

\begin{tabular}{|c|c|c|c|}
\hline \multirow[b]{2}{*}{ Characteristic or treatment } & \multirow[b]{2}{*}{$\begin{array}{c}\text { Run-in phase } \\
(\mathrm{n}=233)\end{array}$} & \multicolumn{2}{|c|}{ Evaluable period } \\
\hline & & $\begin{array}{l}\text { Feedback-off } \\
(n=771)\end{array}$ & $\begin{array}{l}\text { Feedback-on } \\
\quad(\mathrm{n}=815)\end{array}$ \\
\hline \multicolumn{4}{|l|}{ Study site: } \\
\hline King County, Washington & $97(42)$ & $367(48)$ & $432(53)$ \\
\hline Thunder Bay, Ontario & $18(8)$ & $5(1)$ & $38(5)$ \\
\hline Pittsburgh, Pennsylvania & $118(51)$ & $399(52)$ & $345(42)$ \\
\hline Mean (SD) age (years) & $66(17)$ & $66(17)$ & $65(17)$ \\
\hline Male & $152(65)$ & $480(62)$ & $520(64)$ \\
\hline \multicolumn{4}{|l|}{ Cardiac arrest witnessed: } \\
\hline By emergency medical services & $22(9)$ & $82(11)$ & $83(10)$ \\
\hline By known bystander† & 79/211 (37) & 265/689 (38) & 284/732 (39) \\
\hline Bystander CPR $\dagger$ & $101 / 211(48)$ & $347 / 689(50)$ & $383 / 732(52)$ \\
\hline Cardiac arrest in public location & 34 (15) & $98(13)$ & $112(14)$ \\
\hline
\end{tabular}

Mean (SD) response times for emergency

medical services (minutes):

\begin{tabular}{|c|c|c|c|}
\hline From emergency phone call to first arrival & $5.9(2.8)$ & $5.8(2.5)$ & $5.5(2.2)$ \\
\hline From dispatch to shock assessment & $10.6(5.2)$ & $11.4(6.1)$ & $11.7(7.9)$ \\
\hline \multicolumn{4}{|l|}{ First cardiac rhythm recorded: } \\
\hline $\begin{array}{l}\text { Ventricular fibrillation or pulseless } \\
\text { ventricular tachycardiał }\end{array}$ & 45/232 (19) & $182 / 769(24)$ & $191 / 811(24)$ \\
\hline Pulseless electrical activity & $62 / 232(27)$ & $177 / 769(23)$ & $210 / 811(26)$ \\
\hline Asystoleł & 111/232 (48) & $319 / 769(41)$ & $336 / 811(41)$ \\
\hline Other & $14 / 232(6)$ & $91 / 769(12)$ & 74/811 (9) \\
\hline \multicolumn{4}{|l|}{ Prehospital treatment: } \\
\hline >1 shock given $\ddagger$ & $61 / 233(26)$ & $186 / 770(24)$ & $180 / 815(22)$ \\
\hline Mean (SD) No of shocks per case§ & $3.0(0.2)$ & $2.9(0.2)$ & $3.4(0.2)$ \\
\hline Advanced life support on scene & $231(99)$ & $770(100)$ & $813(100)$ \\
\hline Endotracheal intubation & $195(84)$ & $595(77)$ & $646(79)$ \\
\hline Other advanced airway support & $11(5)$ & $96(12)$ & $85(10)$ \\
\hline $\begin{array}{l}\text { Mean (SD) total adrenaline (epinephrine) } \\
\text { dose given (mg) }\end{array}$ & $3.3(2.5)$ & $2.8(2.3)$ & $2.8(2.3)$ \\
\hline
\end{tabular}

*Trial of CPR provided by emergency medical services with monitor-defibrillator providing real-time feedback on CPR process ("feedback-on") or not providing feedback ("feedback-off").

+Percentage of arrests not witnessed by emergency medical services.

$\ddagger$ Percentage of cases not missing data.

$\S$ Among those receiving shocks.

the emergency department and survival to hospital discharge. Analyses of secondary outcomes excluded cases with missing outcome information (the primary outcome, return of spontaneous circulation, was ascertained in all study subjects). Subgroups were defined a priori by presenting rhythm, sex, cause of the arrest, and location of the arrest (public or private). Outcomes analyses were repeated in each of these groups. Treatment group was based on the principle of intention to treat.

Measures of the CPR process were summarised by the mean (standard error) and median (interquartile range) on each treatment arm. The distribution of each CPR variable was estimated by kernel density estimation with a Gaussian kernel function and plotted by treatment arm. CPR variables were compared by randomisation status by fitting a linear regression model that included cluster as a fixed effect. The cluster adjusted difference, 95\% confidence interval for the difference, and $\mathrm{P}$ value are presented. One participating agency randomised individual vehicles. For the analysis of CPR variables, all vehicles from this agency that did not treat at least five subjects during both the feedback-on and feedback-off periods were combined into a super-cluster.

To test for a carryover effect, we examined the treatment effect during the first two treatment periods. Limiting ourselves to those two periods, we calculated the difference in the rates of restoration of spontaneous circulation between the time when defibrillator feedback was off and the time it was on. We then classified clusters as those randomised to feedback-on in the first period or those first randomised to feedback-off. A weighted two sample $t$ test comparing the treatment effect in the two types of clusters was performed as a test for carryover effect. If there was no carryover effect we would expect the average change in cluster rates of return of spontaneous circulation and survival to be similar in those clusters randomised to feedback-on first and those randomised to feedback-off first. This analysis was repeated for the rates of survival to hospital discharge.

\section{RESULTS}

A total of 233 subjects, not included in the final analysis, were enrolled in the run-in phase of the study, during which the monitor-defibrillators were deployed in feedback-off mode, and the results are presented to show the baseline system and population characteristics. Subsequently 1586 consecutive subjects were enrolled (771 initially assigned to feedback-off and 815 to feedback-on) during the cluster-randomised evaluable period and provided the two primary comparison groups (fig 1). No study related adverse events were reported.

Subject demographics, arrest circumstances, and emergency medical services' response intervals did not differ between the groups. Overall, cardiac arrest occurred more commonly among men and in private locations, was witnessed by a bystander or emergency medical services about a third of the time, and most often presented with asystole (table 1).

CPR process information was available for 1174 $(74 \%)$ of the subjects. Compared with the cases with CPR process data, the cases without data were more likely to have been witnessed by emergency medical services, to have arrested in a public location, to have had a first rhythm of ventricular tachycardia or fibrillation or "no shock, no strip available" (when the defibrillator did not fire and no electrocardiogram was available to confirm the initial rhythm), and have had a longer interval between the call to the emergency medical services and their arrival at the scene. Cases without CPR process data were also less likely to have prehospital return of spontaneous circulation. There were no differences between the cases with and without CPR process data in age, sex, bystander witnessed, bystander CPR, return of spontaneous circulation on arrival at the emergency department, or survival to discharge. 
Table 2 | Data on cardiopulmonary resuscitation (CPR) process received by patients with a cardiac arrest outside hospital: 233 patients during run-in phase of trial* and 1586 during evaluable period

\begin{tabular}{|c|c|c|c|c|c|}
\hline & \multirow{2}{*}{$\begin{array}{l}\text { Run-in } \\
\text { period } \\
(n=233)\end{array}$} & \multicolumn{2}{|c|}{ Evaluable period } & \multicolumn{2}{|c|}{ Difference of feedback-on from feedback-off } \\
\hline & & $\begin{array}{l}\text { Feedback-off } \\
\quad(n=771)\end{array}$ & $\begin{array}{l}\text { Feedback-on } \\
(n=815)\end{array}$ & $\begin{array}{l}\text { Cluster adjusted } \\
\text { difference }(95 \% \mathrm{Cl})\end{array}$ & $P$ value \\
\hline \multicolumn{6}{|l|}{ Data for all available minutes } \\
\hline \multicolumn{6}{|l|}{ Minutes of data recorded: } \\
\hline Mean (SE) & $5.5(0.3)$ & $5.6(0.1)$ & $5.5(0.1)$ & $0.1(-0.2$ to 0.5$)$ & 0.481 \\
\hline Median (IQR) & $5(0-10)$ & $6(0-10)$ & $6(0-10)$ & & \\
\hline No of compressions/minute: & $n=166$ & $n=570$ & $n=604$ & & \\
\hline Mean (SE) & $102.9(1.6)$ & $108.0(0.7)$ & $103.1(0.5)$ & $-4.7(-6.4$ to -3.0$)$ & $<0.001$ \\
\hline Median (IQR) & $106(93-116)$ & $109(99-117)$ & $103(96-110)$ & & \\
\hline CPR fraction (\%): & $n=166$ & $n=570$ & $n=604$ & & \\
\hline Mean (SE) & $60.3(1)$ & 64.0 (1) & 65.9 (1) & $1.9(0.4$ to 3.4$)$ & 0.016 \\
\hline Median (IQR) & $61(51-71)$ & $65(56-73)$ & $67(59-74)$ & & \\
\hline Compression depth (mm): & $n=86$ & $n=467$ & $n=529$ & & \\
\hline Mean (SE) & $34.3(1.0)$ & $37.8(0.4)$ & $39.6(0.4)$ & $1.6(0.5$ to 2.7$)$ & 0.005 \\
\hline Median (IQR) & $34(27-42)$ & $37(32-44)$ & $40(35-44)$ & & \\
\hline Compressions with incomplete release (\%): & $n=86$ & $n=467$ & $n=529$ & & \\
\hline Mean (SE) & $25.3(2)$ & $14.6(1)$ & $10.4(1)$ & $-3.4(-5.2$ to -1.5$)$ & $<0.001$ \\
\hline Median (IQR) & $22(7-40)$ & $8(1-22)$ & $5(1-14)$ & & \\
\hline No of ventilations/minute: & $n=24$ & $n=346$ & $n=347$ & & \\
\hline Mean (SE) & $4.9(0.8)$ & $5.6(0.2)$ & $5.8(0.2)$ & $0.1(-0.5$ to 0.7$)$ & 0.727 \\
\hline Median (IQR) & $6(2-7)$ & $5(2-8)$ & $5(3-8)$ & & \\
\hline No (\%) of cases when feedback muted by EMS & $9(5)$ & $6(1)$ & $114(14)$ & & \\
\hline \multicolumn{6}{|l|}{ Data for the first five minutes of resuscitation } \\
\hline No of compressions/minute: & $n=166$ & $n=561$ & $n=595$ & & \\
\hline Mean (SE) & $101.3(2.1)$ & $107.3(0.8)$ & $103.1(0.6)$ & $-3.9(-5.9$ to -2.0$)$ & $<0.001$ \\
\hline Median (IQR) & $106(91-116)$ & $108(98-117)$ & $103(96-111)$ & & \\
\hline CPR fraction (\%): & $n=166$ & $n=562$ & $n=595$ & & \\
\hline Mean (SE) & $58.2(1)$ & 61.9 (1) & $64.1(1)$ & $2.3(0.6$ to 4.1$)$ & 0.008 \\
\hline Median (IQR) & $61(48-71)$ & $63(54-72)$ & $66(56-73)$ & & \\
\hline Compression depth (mm): & $n=81$ & $n=449$ & $n=509$ & & \\
\hline Mean (SE) & $35.6(1.1)$ & $37.7(0.5)$ & $39.7(0.4)$ & 1.7 (0.6 to 2.9$)$ & 0.003 \\
\hline Median (IQR) & $36(29-43)$ & $37(31-45)$ & $40(35-45)$ & & \\
\hline Compressions with incomplete release (\%): & $n=81$ & $n=448$ & $n=509$ & & \\
\hline Mean (SE) & $25.4(3)$ & $14.6(1)$ & 10.9 (1) & $-2.9(-4.9$ to -0.8$)$ & 0.007 \\
\hline Median (IQR) & $17(2-47)$ & $7(1-22)$ & $4(1-14)$ & & \\
\hline No of ventilations/minute: & $n=22$ & $n=335$ & $n=335$ & & \\
\hline Mean (SE) & $4.3(0.9)$ & $4.9(0.2)$ & $5.2(0.2)$ & $0.2(-0.4$ to 0.8$)$ & 0.501 \\
\hline Median (IQR) & $4(1-6)$ & $4(2-7)$ & $5(2-7)$ & & \\
\hline
\end{tabular}

The median (interquartile range) time of CPR process information available for analysis was $6(0-10)$ minutes. In the feedback-on arm, emergency medical service providers muted the audible feedback in 114 $(14 \%)$ of the cases. During all available minutes of data (table 2), cases randomised to feedback-on compared with feedback-off had significantly lower mean compression rate (103 v 108 per minute, $\mathrm{P}<0.001$ ), higher chest compression fraction $(66 \%$ v 64\%, $\mathrm{P}=0.016)$, deeper chest compressions (40 v $38 \mathrm{~mm}$, $\mathrm{P}=0.005)$, and fewer chest compressions with incomplete release $(10 \% v 15 \%, \mathrm{P}<0.001)$. There were no significant differences between groups for number of ventilations per minute, proportion of patients receiving more than one shock, or mean number of shocks per case. Similar relationships were evident when only the first five minutes of data were examined (table 2).

A descriptive examination of the distribution of the CPR process variables (fig 2) suggests that provision of real-time feedback resulted in more consistent compression rate and compression depth and fewer compressions with incomplete release, with the feedbackon group having a narrower frequency distribution than the feedback-off group and smaller standard deviations in each of the plots.

During the evaluation period, the proportion of patients with prehospital return of spontaneous 

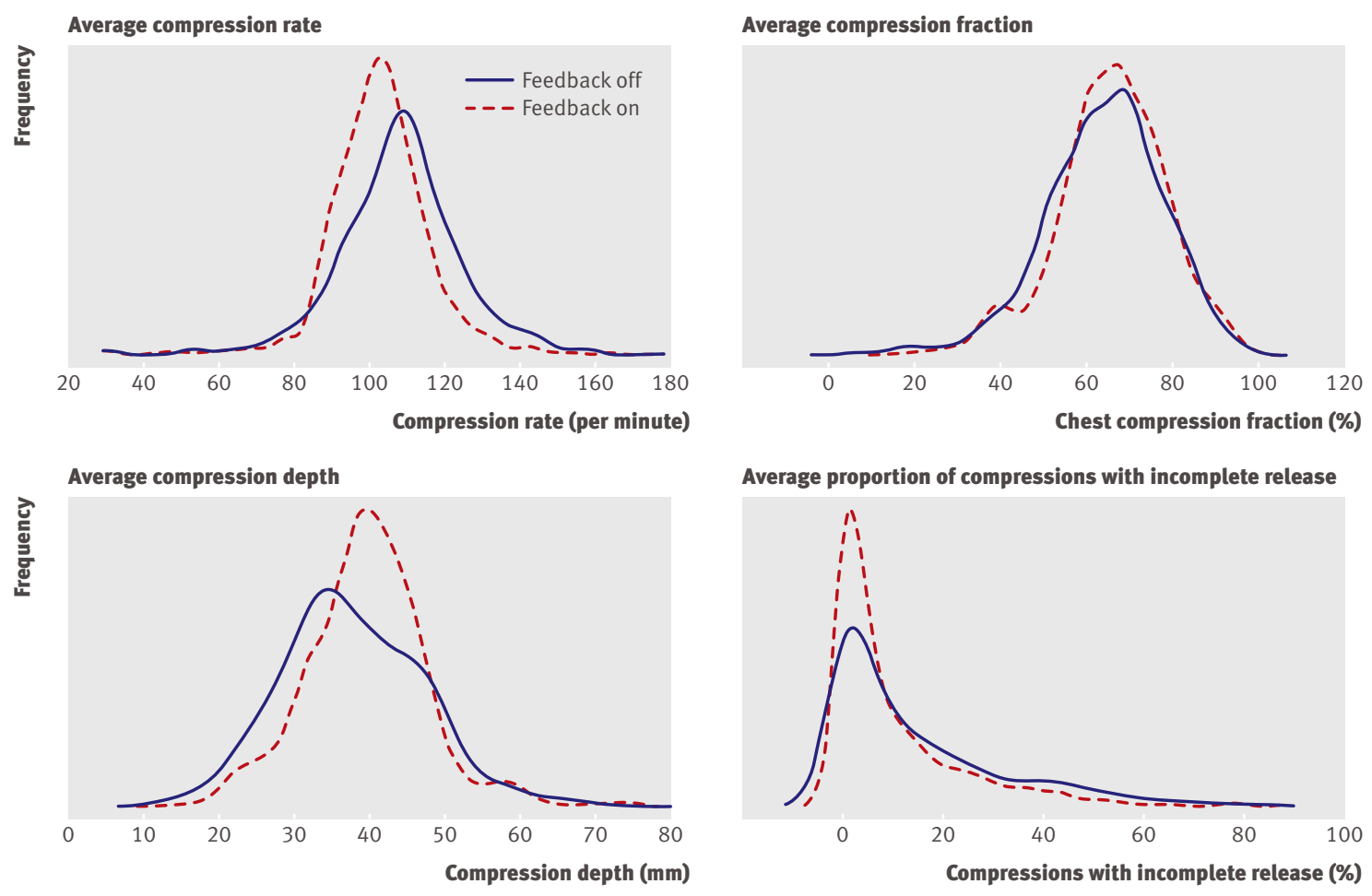

Fig 2 | Frequency distribution of the rate, fraction, and depth of chest compressions and the percentage of chest compressions with incomplete release during cardiopulmonary resuscitation stratified by whether monitor-defibrillators provided real-time feedback ("feedback-on") or not ("feedback-off")

circulation (primary outcome) did not significantly differ between the feedback-on group (361/815 patients $(44 \%))$ and feedback-off group (345/771 (45\%)) (table 3). Similarly, presence of a pulse at the emergency department, survival to hospital discharge, and discharge awake did not significantly differ by feedback status (table 3).

Subgroup comparisons, planned a priori, by sex and cause of cardiac arrest (presumed cardiac origin $v$ not) did not reveal differences in outcomes between the two study groups. However, analysis by first documented electrocardiographic rhythm showed that, among those with an initial electrocardiogram rhythm of asystole, a lower proportion of patients in the feedback-on group had a pulse at arrival in the emergency department than in the feedback-off group (43 (13\%) v 59 $(18 \%), \mathrm{P}=0.029)$ (table 4). Hospital treatments for the patients surviving at least one day and the discharge status for those surviving to hospital discharge were similar for both feedback-off and feedback-on groups (table 5).

Table 6 presents the analysis for possible carryover effects by comparing the clusters first randomised to feedback-on with those clusters first randomised to feedback-off during the first two randomisation periods. There was no evidence for a carryover effect. In a retrospective analysis, we did note an apparent outcome difference between feedback-off and feedbackon based on the randomisation period. Feedback-off during these first two randomisation periods was associated with a greater likelihood of survival than feedback-on (15\% v 10\%, cluster adjusted average difference -5.5 (95\% confidence interval -9.6 to -1.3$)$ ). However, for subsequent randomisation periods, feedback-on was associated with a greater likelihood of survival $(13 \%$ v 10\%, cluster adjusted average difference $1.6(-3.1$ to 6.4$))$. A similar relation was observed for the restoration of spontaneous circulation outcome, whereby feedback-off was associated with greater likelihood of restoration of spontaneous circulation during the first two randomisation periods and feedback-on was associated with greater likelihood of restoration of spontaneous circulation during subsequent randomisation periods.

\section{DISCUSSION}

This randomised controlled trial among people having a cardiac arrest outside hospital compared performance and outcomes of cardiopulmonary resuscitation for subjects who were treated with or without real-time audio and visual CPR feedback. The proportion of patients with return of spontaneous circulation did not significantly differ between feedback-on (44\%) and feedback-off (45\%) groups. Similarly, the secondary outcomes of the presence of a pulse at arrival at the emergency department, survival to hospital discharge, and neurological status at discharge did not significantly differ by assigned feedback status. Providing real-time feedback did result in CPR performance closer to the established resuscitation guidelines.

During cardiac arrest, CPR can help sustain left ventricular filling, prevent right ventricular distension, and 
Table 3 |Clinical outcomes of patients receiving cardiopulmonary resuscitation (CPR) outside hospital: 233 patients during run-in phase of trial and 1586 during evaluable period*. Values are numbers (percentages) of patients unless stated otherwise

\begin{tabular}{|c|c|c|c|c|c|}
\hline \multirow[b]{2}{*}{ Outcome } & \multirow[b]{2}{*}{$\begin{array}{l}\text { Run-in phase } \\
\quad(n=233)\end{array}$} & \multicolumn{2}{|c|}{ Evaluable period } & \multicolumn{2}{|c|}{ Difference of feedback-on from feedback-off } \\
\hline & & $\begin{array}{l}\text { Feedback-off } \\
\qquad(n=771)\end{array}$ & $\begin{array}{l}\text { Feedback-on } \\
\qquad(n=815)\end{array}$ & $\begin{array}{l}\text { Average change in cluster } \\
\text { percentages }(95 \% \mathrm{Cl})\end{array}$ & $P$ value \\
\hline Prehospital return of spontaneous circulation & $106(45)$ & $345(45)$ & $361(44)$ & $0.1(-4.4$ to 4.6$)$ & 0.962 \\
\hline $\begin{array}{l}\text { Transported to emergency department or } \\
\text { hospital }\end{array}$ & $156(67)$ & $438(57)$ & $461(57)$ & $-1.6(-4.5$ to 1.4$)$ & 0.298 \\
\hline With pulse at arrival at emergency department & $72(31)$ & $243(32)$ & $260(32)$ & $-0.8(-4.9$ to 3.4$)$ & 0.713 \\
\hline Survival for $\geq 1$ day & $65(28)$ & $213(28)$ & $234(29)$ & $0.4(-4.6$ to 5.4$)$ & 0.862 \\
\hline Survival to hospital discharge & $27(12)$ & $96(12)$ & $92(11)$ & $-1.5(-3.9$ to 0.9$)$ & 0.206 \\
\hline Discharge from hospital awake & $20(9)$ & $78(10)$ & $84(10)$ & $-0.2(-2.5$ to 2.1$)$ & 0.855 \\
\hline
\end{tabular}

*Trial of CPR provided by emergency medical services with monitor-defibrillator providing real-time feedback on CPR process ("feedback-on") or not providing feedback ("feedback-off").

maintain a measure of systemic arterial perfusion to the brain and heart. ${ }^{15}$ Early bystander CPR can improve the likelihood of resuscitation. Several experimental studies have indicated that the specific composition, timing, and quantity of CPR (chest compressions and ventilations) can influence the likelihood of successful resuscitation, suggesting that the content of CPR may be modified or refined to improve survival. ${ }^{31617}$ To this end, major systematic changes in prehospital CPR performance have been associated with important advances in survival, providing support for the concept that the characteristics of CPR can affect outcome. ${ }^{18-20}$ In general, these protocol-level changes have increased the quantity of chest compressions and, in turn, limited delays and interruptions in CPR.

\section{Comparisons with other studies}

Real-time feedback potentially helps improve CPR by prompting providers to deliver the various components of resuscitation (such as depth and rate of chest compressions) as close to the established guidelines as possible. The Q-CPR software used in this study and the related software in the voice advisory training manikin have been studied in training, simulated resuscitation, and field conditions. For training, the voice advisory manikin software improves skill acquisition and retention in both lay public and prehospital care providers. ${ }^{2122}$ However, use of the voice advisory software to guide CPR performance by nurses, field emergency medical service providers, and paramedic students on a manikin has typically limited the effects to increasing chest compression depth. ${ }^{23-25}$

Two observational studies, one prehospital and one in hospital, examined the association between realtime feedback and clinical outcomes. The prehospital investigation observed an increase in chest compression depth and a reduction in chest compression rate associated with feedback. ${ }^{7}$ The study in hospital observed no change in CPR measures associated with feedback. ${ }^{8}$ Neither study randomised the intervention nor observed a difference in clinical outcome associated with feedback, but neither study was adequately powered to detect a clinically important effect.

Our study randomised the feedback intervention in more than 1500 cardiac arrest cases and was sufficiently powered to detect a change in prehospital

Table $4 \mid$ Clinical outcomes of patients receiving cardiopulmonary resuscitation (CPR) outside hospital during evaluable period of trial* by initial cardiac rhythm recorded. Values are numbers (percentages) of patients unless stated otherwise

\begin{tabular}{|c|c|c|c|c|}
\hline \multirow[b]{2}{*}{ Initial cardiac rhythm and clinical outcomes } & \multicolumn{2}{|c|}{ Evaluable period } & \multicolumn{2}{|c|}{ Difference of feedback-on from feedback-off } \\
\hline & Feedback-off & Feedback-on & $\begin{array}{c}\text { Cluster adjusted difference in } \\
\text { percentage }(95 \% \mathrm{Cl})\end{array}$ & $P$ value \\
\hline Ventricular fibrillation or pulseless ventricular tachycardia: & $n=182$ & $n=191$ & & \\
\hline Return of spontaneous circulation (any) & $121(66)$ & $129(68)$ & $0.7(-8.0$ to 9.5$)$ & 0.861 \\
\hline Pulse at arrival at emergency department & $96(53)$ & $106(55)$ & $0.6(-11.2$ to 12.4$)$ & 0.910 \\
\hline Survival to hospital discharge & $64(35)$ & $60(31)$ & $-5.1(-15.8$ to 5.6$)$ & 0.325 \\
\hline Pulseless electrical activity: & $\mathrm{n}=177$ & $n=210$ & & \\
\hline Return of spontaneous circulation (any) & $95(54)$ & $101(48)$ & $-6.8(-17.1$ to 3.4$)$ & 0.170 \\
\hline Pulse at arrival at emergency department & $67(38)$ & $84(40)$ & $0.2(-10.8$ to 11.2$)$ & 0.969 \\
\hline Survival to hospital discharge & $18(10)$ & $24(11)$ & $1.2(-5.1$ to 7.5$)$ & 0.688 \\
\hline Asystole: & $n=319$ & $n=336$ & & \\
\hline Return of spontaneous circulation (any) & $91(29)$ & $91(27)$ & $0.7(-4.2$ to 5.7$)$ & 0.769 \\
\hline Pulse at arrival at emergency department & $59(18)$ & $43(13)$ & $-5.5(-10.5$ to -0.6$)$ & 0.029 \\
\hline Survival to hospital discharge & $8(3)$ & $4(1)$ & $-1.2(-2.5$ to 0.2$)$ & 0.092 \\
\hline
\end{tabular}

*Trial of CPR provided by emergency medical services with monitor-defibrillator providing real-time feedback on CPR process ("feedback-on") or not providing feedback ("feedback-off"). 
Table $5 \mid$ Hospital treatments and status at hospital discharge among patients receiving cardiopulmonary resuscitation (CPR) outside hospital during evaluable period of trial*. Values are numbers (percentages $\dagger$ ) of patients

\begin{tabular}{lcc} 
& \multicolumn{2}{c}{ Evaluable period } \\
\cline { 2 - 3 } Hospital treatments in patients surviving $\geq 1$ day & Feedback-off & Feedback-on \\
\hline Hypothermia & $\mathrm{n}=\mathbf{2 1 3}$ & $\mathrm{n}=\mathbf{2 3 4}$ \\
\hline Diagnostic catheterisation & $117(57)$ & $130(57)$ \\
\hline Percutaneous coronary intervention & $50(24)$ & $53(23)$ \\
\hline Coronary artery bypass graft surgery & $43(21)$ & $35(15)$ \\
\hline Permanent pacemaker & $5(2)$ & $7(4)$ \\
\hline Implantable cardioverter defibrillator & $4(2)$ & $19(8)$ \\
\hline Status of subjects surviving to hospital discharge & $23(11)$ & $\mathrm{n}=92$ \\
\hline Awake, no disability reported & $\mathrm{n}=96$ & $35(39)$ \\
\hline Awake with presumed disability, discharged home & $29(32)$ & $13(15)$ \\
\hline Awake with presumed disability, discharged to inpatient rehab & $12(13)$ & $15(17)$ \\
\hline Awake with presumed disability, discharged to nursing home & $22(24)$ & $18(20)$ \\
\hline Not awake & $15(16)$ & $8(9)$ \\
\hline Missing discharge status/location & $5(5)$ & $3(3)$ \\
\hline
\end{tabular}

*Trial of CPR provided by emergency medical services with monitor-defibrillator providing real-time feedback on CPR process ("feedback-on") or not providing feedback ("feedback-off").

†Data missing in approximately $3 \%$ of cases, and percentages based on non-missing data.

return of spontaneous circulation of $10 \%$. The intervention did produce chest compressions that were on average $2 \mathrm{~mm}$ deeper (38 $v 40 \mathrm{~mm}$ ), five compressions per minute slower (108 v 103), more likely to have complete release $(85 \%$ v $90 \%)$, and less likely to be interrupted or delayed (chest compression fraction of 64\% v 66\%). However, these changes in CPR process did not translate to improvements in clinical outcomes.

\section{Strengths and limitations of the study}

A variety of explanations may account for this lack of effect on survival. Firstly, feedback induced changes in CPR performance may be too small to affect outcome. This and other studies indicate that feedback can modify CPR performance; and CPR performance has been associated with outcome. ${ }^{5}$ Nevertheless, larger changes in CPR may be required to alter survival. Improvements in the content, delivery, and interface of real-time feedback may enable greater changes in CPR performance.

Secondly, the influence of feedback on outcome may be related to baseline CPR performance. Feedback may have a greater potential impact in settings where CPR performance is poorest. In our study the CPR measures achieved in the feedback-off arm were better than previously reported for outside hospital, leaving little opportunity for improvement by realtime feedback.

Thirdly, the current CPR guidelines that are encouraged by real-time feedback may not be optimal. For example, feedback lowered the average compression rate from 108 to 103 , consistent with guideline recommendations, but there is no evidence that a rate of 100 per minute is superior to 110 per minute. Indeed, a higher rate would produce more total chest compressions and might produce better circumstances for resuscitation. The ability for feedback to improve outcomes is only as good as the understanding used to inform the CPR guidelines.

Fourthly, the style of feedback tested in this study was corrective (that is, input was provided only when CPR performance did not meet guidelines) as opposed to prescriptive (such as a continuous metronome). It is possible that the feedback distracted from other aspects of resuscitation, offsetting any benefit. Other forms of feedback or the different styles in which feedback is delivered may produce different results.

This trial has several potential limitations. Providers could not be blinded to the intervention so that their care could have been influenced by their perceptions of the merit of feedback. Awareness that CPR performance was being monitored might have produced a Hawthorne effect regardless of intervention arm, making it more difficult to show a difference. ${ }^{26}$ Indeed, measures of CPR complied more closely with guidelines during the evaluable phase compared with the run-in phase for both feedback-on and feedback-off arms.

We cannot rule out the possibility of a training effect in clusters reassigning from feedback-off to feedbackon. However, given that the treatment of cardiac arrest by an individual prehospital care provider occurs infrequently, the likelihood of two cases treated by an individual provider around the time of reassignment to the other treatment arm is small.

The emergency medical services participating in this study may not be typical of agencies across North America. Although diverse in both operational practices and population served, agencies with the resources and commitment required to participate in prehospital clinical trials may deliver a different level of care from those that do not. ${ }^{27} \mathrm{We}$ made no corrections for the multiple comparisons made between feedback-on and feedback-off. These limitations are offset

Table 6 |Tests for carryover effect by comparison of the clusters of cardiopulmonary resuscitations (CPR) outside hospital first randomised to feedback-on with those clusters first randomised to feedback-off during the first two randomisation periods of trial*

Difference of feedback-on from feedback-off Average percentage change in cluster outcome rates $(95 \% \mathrm{Cl})$

Outcome

Prehospital return of spontaneous circulation Survival to hospital discharge

Feedback-on first clusters Feedback-off first clusters

$-8.4(-17.4$ to 0.7$)$

$-5.6(-11.9$ to 0.8$)$

$-7.3(-17.5$ to 2.9$)$

$-5.3(-11.7$ to 1.0$)$
$P$ value for test of carryover 0.865 0.959

*Trial of CPR provided by emergency medical services with monitor-defibrillator providing real-time feedback on CPR process ("feedback-on") or not providing feedback ("feedback-off') 


\section{WHAT IS ALREADY KNOWN ON THIS TOPIC}

The composition, timing, and quantity of cardiopulmonary resuscitation (chest compressions and ventilations) can influence the likelihood of successful resuscitation

Real time cardiopulmonary feedback has been shown to improve short term outcomes in some emergency medical service systems

\section{WHAT THIS STUDY ADDS}

Provision of real-time feedback during cardiopulmonary resuscitation by emergency medical services resulted in resuscitation performance closer to established guidelines

However, the feedback did not improve patients' survival to hospital discharge

by the study's strengths - a randomised trial design with rigorous data collection procedures within a well developed clinical trial group.

The study evaluated one style of feedback. It is possible that the current version of feedback improved some parts but distracted from other aspects of resuscitation, offsetting any benefit. Interestingly, retrospective analysis suggested feedback-on was associated with lower likelihood of favourable outcome during the first two randomisation periods and a greater likelihood of successful resuscitation for subsequent randomisation periods. These results may be due to chance, but other potential explanations include the presence of a learning curve, whereby the feedback became more effective as providers gained experience with the interface. Other feedback modalities or content may produce different results.

\section{Conclusions}

Provision of real-time feedback during CPR by emergency medical services did not alter the proportion of subjects achieving return of spontaneous circulation in the field even though CPR performance was closer to established guidelines when feedback was provided. It is likely that successful resuscitation requires an integrated and timely set of actions, of which CPR is a core component. Feedback provides a strategy to alter individual CPR performance, although the current feedback alone did not improve outcome. Ultimately the role of feedback may be determined by our understanding of cardiac arrest and resuscitation physiology coupled with efforts to optimise the effectiveness of CPR feedback.

Contributors: All authors contributed to the final study design, data interpretation, and manuscript revision. DH and CWC implemented the study in the Pennsylvania sites, TDR and PJK implemented the study in Washington. IGS implemented the study in Ontario. SE-S, SSE, and GN wrote the statistical analysis plan and performed the analyses at the end of subject enrolment. DH drafted the manuscript and coordinated subsequent revisions. DH is guarantor of the work, but all authors had full access to the data and can take responsibility for their integrity and the accuracy of the data analysis.

Funding: The Resuscitation Outcomes Consortium is supported by a series of cooperative agreements with 10 regional clinical centres and one data coordinating centre (5U01 HL077863, HL077881, HL077871 HL077872, HL077866, HL077908, HL077867, HL077885, HL077887, HL077873, HL077865) from the National Heart, Lung and Blood Institute in partnership with the National Institute of Neurological Disorders and Stroke, US Army Medical Research \& Materiel Command, the Canadian Institutes of Health Research (CIHR)-Institute of Circulatory and
Respiratory Health, Defence Research and Development Canada, the Heart and Stroke Foundation of Canada, and the American Heart Association. The monitor-defibrillators used in the study were donated to participating emergency medical services by Philips Healthcare, Andover, Massachusetts, USA, with support from Laerdal Medical AS, Stavanger, Norway. The sponsors had no role in the design and conduct of the study or in the collection, management, or analysis of the data. The sponsors were provided an opportunity to make non-binding comments on the manuscript before submission.

Competing interests: All authors have completed the Unified Competing Interest form at www.icmje.org/COI_disclosure.pdf (available on request from the corresponding author) and declare that 1) all authors have support from the listed funding agencies for the submitted work; 2) financial relationships with companies that might have an interest in the submitted work (DH, CWC, TDR, and GN received funding from Laerdal Medical or the Laerdal Foundation for Acute Medicine, CWC holds a patent related to defibrillation currently licensed to Medtronic ERS, TDR has received funding from Philips Healthcare, GKS holds stock in the Medtronic Corporation and is the inventor on two patents related to implantable defibrillators, assigned to Boston Scientific, GN has received research funding from Baxter and the Medtronic Foundation and is a research collaborator with Gambro Renal (Lakewood, CO), Sotera Wireless (San Diego, CA), and Lifebridge Medizintechnik AG (Ampfing, Germany), GN and CWC are compensated by the American Heart Association as committee chair, co-chair, member of affiliate board of directors, or worksheet reviewer); 3) no other relationships or activities that could appear to have influenced the submitted work.

Ethical approval: The study was approved by the local institutional review or research ethics boards for each participating agency, with waiver of informed consent under minimal risk criteria.

Data sharing: A copy of the full trial protocol can be obtained from the University of Washington Clinical Trials Center.

1 Wik L, Kramer-Johansen J, Myklebust H, Sorebo H, Svensson L, Fellows $B$, et al. Quality of cardiopulmonary resuscitation during outof-hospital cardiac arrest. JAMA 2005;293:299-304.

2 Abella BS, Alvarado JP, Myklebust H, Edelson DP, Barry A, O'Hearn N, et al. Quality of cardiopulmonary resuscitation during in-hospital cardiac arrest. JAMA 2005;293:305-10.

3 Aufderheide T, Sigurdsson G, Pirrallo RG, Yannopoulos D, McKnite S, von Briesen C, et al. Hyperventilation-induced hypotension during cardiopulmonary resuscitation. Circulation 2004;109:1960-5.

4 Berg RA, Sander AB, Kern KB, Hilwig RW, Heidenreich JW, Porter ME, et al. Adverse hemodynamic effects of interupting chest compressions for rescue breathing during cardiopulmonary resuscitation for ventricular fibrillation cardiac arrest. Circulation 2001;104:2465-70.

5 Christenson J, Andrusiek D, Everson-Stewart S, Kudenchuk P, Hostler D, Powell J, et al. Chest compression fraction determines survival in patients with out-of-hospital ventricular fibrillation. Circulation 2009;120:1241-7.

6 Yeung J, Meeks R, Edelson D, Gao F, Soar J, Perkins GD. The use of CPR feedback/prompt devices during training and CPR performance: a systematic review. Resuscitation 2009;80:743-51.

7 Kramer-Johansen J, Myklebust H, Wik L, Fellows B, Svensson L, Sorebo H, et al. Quality of out-of-hospital cardiopulmonary resuscitation with real time automated feedback: a prospective interventional study. Resuscitation 2006;71:283-92.

8 Abella BS, Edelson DP, Kim S, Retzer E, Myklebust H, Barry AM, et al. CPR quality improvement during in-hospital cardiac arrest using a real-time audiovisual feedback system. Resuscitation 2007;73:54-61.

9 Morrison LJ, Nichol G, Rea TD, Christenson J, Callaway CW, Stephens S, et al. Rationale, development and implementation of the Resuscitation Outcomes Consortium Epistry-Cardiac Arrest. Resuscitation 2008;78:161-9.

10 Nichol G, Thomas E, Callaway CW, Hedges J, Powell JL, Aufderheide TP, et al. Regional variation in out-of-hospital cardiac arrest incidence and outcome. JAMA 2008;300:1423-31.

11 Aufderheide TP, Kudenchuk PJ, Hedges JR, Nichol G, Kerber RE, Dorian P, et al. Resuscitation Outcomes Consortium (ROC) PRIMED cardiac arrest trial methods part 1: rationale and methodology for the impedance threshold device (ITD) protocol. Resuscitation 2008;78:179-85.

12 Stiell IG, Callaway C, Davis D, Terndrup T, Powell J, Cook A, et al. Resuscitation Outcomes Consortium (ROC) PRIMED cardiac arrest trial methods part 2: rationale and methodology for "analyze later vs analyze early" protocol. Resuscitation 2008;78:186-95.

13 Macdonald RD, Swanson JM, Mottley JL, Weinstein C. Performance and error analysis of automated external defibrillator use in the out of-hospital setting. Ann Emerg Med 2001;38:262-7. 
14 Turner R, White I, Croudace T. Analysis of cluster randomized crossover trial data: a comparison of methods. Stat Med 2007;26:274-89.

15 Rea TD, Cook AJ, Hallstrom A. CPR during ischemia and reperfusion: a model for survival benefits. Resuscitation 2008;77:6-9.

16 Berg RA, Hilwig RW, Berg MD, Berg DD, Samson RA, Indik JH, et al. Immediate post-shock chest compressions improve outcome from prolonged ventricular fibrillation. Resuscitation 2008;78:71-6.

17 Tang W, Snyder D, Wang J, Huang L, Chang YT, Sun S, et al. One-shock versus three-shock defibrillation protocol significantly improves outcome in a porcine model of prolonged ventricular fibrillation cardiac arrest. Circulation 2006;113:2683-9.

18 Bobrow BJ, Clark LL, Ewy GA, Chikani V, Sanders AB, Berg RA, et al. Minimally interrupted cardiac resuscitation by emergency medical services for out-of-hospital cardiac arrest. JAMA 2008;299:1158-65.

19 Rea TD, Helbock M, Perry S, Garcia M, Cloyd D, Becker L, et al. Increasing use of cardiopulmonary resuscitation during out-ofhospital ventricular fibrillation arrest: survival implications of guideline changes. Circulation 2006;114:2760-5.

20 Sayre MR, Cantrell SA, White LI, Hiestand BC, Keseg DP, Koser S. Impact of the 2005 American Heart Association cardiopulmonary resuscitation and emergency cardiovascular care guidelines on outof-hospital cardiac arrest survival. Prehosp Emerg Care 2009;13:469-77.

21 Sutton RM, Donoghue A, Myklebust H, Srikantan S, Byrne A, Priest M, et al. The voice advisory manikin (VAM): an innovative approach to pediatric lay provider basic life support skill education. Resuscitation 2007;75:161-8.

22 Wik L, Myklebust H, Auestad BH, Steen PA. Retention of basic life support skills 6 months after training with an automated voice advisory manikin system without instructor involvement. Resuscitation 2002;52:273-9.

23 Dine CJ, Gersh RE, Leary M, Riegel BJ, Bellini LM, Abella BS. Improving cardiopulmonary resuscitation quality and resuscitation training by combining audiovisual feedback and debriefing. Crit Care Med 2008;36:2817-22.

24 Hostler D, Wang H, Parrish K, Platt TE, Guimond G. The effect of a voice assist manikin (VAM) system on CPR quality among prehospital providers. Prehosp Emerg Care 2005;9:53-60.

25 Handley AJ, Handley SAJ. Improving CPR performance using an audible feedback system suitable for incorporation into an external defibrillator. Resuscitation 2003;57:57-62.

26 Campbell JP, Maxey VA, Watson WA. Hawthorne effect: implications for prehospital research. Ann Emerg Med 1995;26:590-4.

27 Davis DP, Garberson LA, Andrusiek DL, Hostler D, Daya M, Pirrallo R, et al. A descriptive analysis of Emergency Medical Service Systems participating in the Resuscitation Outcomes Consortium (ROC) network. Prehosp Emerg Care 2007;11:369-82.

Accepted: 2 November 2010 